\section{Thermodynamics without tears}

\section{Tony Guénault}

Thermodynamics and an Introduction to Thermostatistics, 2nd Edn. By Herbert B. Callen. Wiley:1985. Pp.493. \$45.15, £34.75.

The Theory of Thermodynamics. By J.R. Waldram. Cambridge University Press: 1985. Pp.336. Hbk £30, \$59.50; pbk $£ 10.95, \$ 24.95$.

BY AND large, bed-time reading and thermodynamics do not go together. But here are two remarkably unrepresentative examples among the ensemble of thermodynamic textbooks.

Callen's classic Thermodynamics was first published in 1960, and well-thumbed copies are still to be found in every library. The fact that no revision has been necessary in the intervening 25 years speaks for itself, and the main change in this second edition is the inclusion of a compact section on thermostatistics (for want of a better word!). Callen's logic is that the macro- and micro-approaches to thermodynamics are not so distinct that a modern text should cover only one of them, but that the structure of either discipline should not be lost in the vague blanket coverage of some so-called thermal physics. The statistical method is introduced with admirable clarity through the treatment of an Einstein model of a solid. There are welcome additions on critical phenomena and quantum fluids, the discussions of irreversible thermodynamics and fluctuations have been reorganized, and many new and useful problems are included.

At first glance, the title of Waldram's book does not seem too appealing. However, "theory" here in no way means an obsession with computational detail, but rather a desire to expound clearly the underlying logical structure of the subject. Students are encouraged to investigate the necessary "applications" in problems at the end of each chapter. This is a most impressive piece of work - a thorough, scholarly and original contribution to the teaching of physics.

The unusual starting-point chosen by Waldram is a somewhat intuitive treatment of kinetics and the master equation. This then persuasively leads to expositions of statistical and classical thermodynamics, which are developed distinctly but from the same underlay. The whole text is beautifully graded, starting at a reasonably elementary (first-year university) level, but progressing in the later chapters to topics and ideas which, although immediately accessible to a physics graduand, are beyond the practical scope of most third-year physics courses. An indication of the breadth is that among these topics are transport properties in metals, the law of mass action, imperfect gases and liquids, and graining and density matrices

The clear structure of the book will enable it to be used for a variety of short courses, and at several levels. The reader who actually reads the preface and then the index will unearth three possible subsets of the coverage as a whole - one on classical thermodynamics, one comprising temperature, Boltzmann factors and kinetic theory, and one on chemical thermodynamics; incidentally, one wonders why a more accessible (diagrammatic?) method was not found for displaying this excellent feature. Otherwise the presenta-

tion is admirable in every respect; the pages are large and uncluttered, with useful marginal notes, and a complete index is provided.

These are two particularly wellstructured and clearly written works. The stress of each of them is on the basic logic of the subject, each is satisfyingly successful and yet the solutions reached by the two authors are quite different. Any teacher or researcher will therefore have good reason for buying both books. But I suspect that many a student (with a pocket for only the one) will appreciate having Waldram's book to hand (by the bed, perhaps?) for years to come.

Tony Guénault is a Reader in the Department of Physics, University of Lancaster, Lancaster $L A 14 Y B, U K$

\section{Relative approaches}

\section{D.J. Raine}

Basic Concepts in Relativity and Early Quantum Theory, 2nd Edn. By Robert Resnick and David Halliday. Wiley: 1985. Pp.341. \$34.95, £36.95.

Elements of Relativity Theory. By D. F. Lawden. Wiley: 1985. Pp.108. Pbk £4.95, $\$ 7.95$

RELATIVITY and quantum theory provide interesting contrasts. Together they form the basis of modern physics, but the approach to the teaching of them has traditionally been very different.

With relativity one can go almost straight to the basic postulates, and the essential equations can be mastered with elementary algebra. But, despite references to "high gamma" engineering, there is not a lot to which one can practically apply the resulting knowledge of special relativity, on its own, at least. On the other hand, quantum theory is traditionally approached through several levels, some of which, like the Bohr theory and the more problematical aspects of waveparticle duality, have to be discarded before a complete understanding can be reached - if, indeed, the theory is completely comprehensible at all. Elementary introductions here tend to fall rather short in covering the abundance of realistic practical application. Furthermore, in teaching relativity the arguments historically used in the development of the theory can be employed. In approaching quantum theory the appropriate background in classical Hamiltonian mechanics cannot be assumed, and only the old quantum theory is available to bridge the gap.

The book by Resnick and Halliday divides along these traditional lines. What unifies it is the authors' clear vision of their audience, and an attention to detail facilitated by a second edition. We are told that the authors have also written the most widely used physics textbook in history. I can quite believe it. Certainly, for its wide range of interesting problems and flexibility of organization one can forgive the occasional false bonhomie in references to Sally and Steve and their relatives; this book is an excellent text for a first-year introductory course.

Professor Lawden also knows his intended audience; his Elements of Relativity Theory grew out of lectures for science and engineering students not specializing in theoretical physics, and is now offered for more general consumption. The book gives a traditional, workmanlike account of the Lorentz transformations, using the $\mathrm{k}$-calculus, and of relativistic mechanics, with occasional side-swipes at positivism (which he actually conflates with phenomenalism) and at popular misexpositions. But it is indicative of the problems of this multi-level approach that an appendix deals with the derivative of $x^{2}$ (presumably in order to substantiate the claim that the only pre-requisite is elementary algebra), while a few pages earlier the geodesic equations have been written out.

Both books end with short introductions to general relativity which could scarcely be more different. In fact, it is now clear that the so-called geodesic principle, and the introduction of Riemannian geometry, are not features distinct from the equivalence principle; the gravitational redshift follows directly from the equivalence principle, not from the full theory, whereas the opposite is the case for the bending of light. And while the experimental tests of the theory may not be numerous, they are meagre only if here, too, one ignores recent developments. Needless to say, perhaps, Resnick and Halliday do not.

$D$.J. Raine is a Lecturer in the Department of Astronomy, University of Leicester, Leicester LE1 TRH, UK 